\title{
Spontaneous Subcutaneous and Muscular Bleeding due to Acquired Hemophilia A in Association with Poorly-Controlled Asthma: A Case Report
}

\section{Askin $\mathrm{D}^{1 *}$, Baskin $\mathrm{M}^{2}$ and Levine $\mathrm{RL}^{3}$}

${ }^{1}$ Lenox Hill Hospital, Department of Hematology and Oncology, NY, USA

${ }^{2}$ St Lukes-Roosevelt Hospital, Department of Pulmonary Medicine, NY, USA

${ }^{3}$ Lenox Hill Hospital, Director of Blood Bank, NY, USA

Acquired hemophilia $\mathrm{A}$ is due to the development of autoantibodies to factor VIII, which can occur spontaneously in healthy individuals or secondary to autoimmune disorders, drugs, malignancy, or the post-partum period. It has also been described in the literature to develop in association with asthma. Given the high incidence of asthma, healthcare providers should be aware of the association between this common condition and the much less common acquired factor VIII deficiency, a serious bleeding disorder with significant morbidity.

The presentation of patients acquiring factor VIII deficiency will depend on the level of the inhibitor present, which is inversely proportional to the percentage of active factor VIII available for hemostasis. This paper describes a patient with poorly controlled asthma who acquired a factor VIII inhibitor at the age of 70 .

\section{Case}

The patient is a 70 year old Hispanic female with a past medical history significant for hypertension, GERD, osteoarthritis, and poorly controlled asthma, including a hospitalization for an asthmatic exacerbation eight weeks prior to her diagnosis of acquired hemophilia A. She reported no personal or family history of bleeding disorders or autoimmune disease. Her medications at the time of presentation included amlodipine, montelukast, baclofen, sucralfate, prednisone taper, albuterol, fluticasone/salmeterol, zolpidem, and esomeprazole. She reported no known drug allergies.

Her asthma had been well-controlled on monteleukast $10 \mathrm{mg}$ daily and fluticasone/salmeterol 250/50 mcg twice daily through her adult life until approximately one year prior to the development of her factor VIII inhibitor. She required three separate admissions over the year and required oral prednisone for the majority of the year. Endoscopy and ENT examination revealed severe gastroesophageal and laryngeal reflux. Her omeprazole was changed to high dose esomeprazole. A chest CT and laboratory evaluation ruled out allergic bronchopulmonary aspergillosis and vasculitis.

She was referred for hematology consultation because of spontaneous subcutaneous bleeding and bruising of her upper and lower extremities, with the largest ecchymotic area extending from her right groin to her knee. Her initial labs were significant for an aPTT of 100.6 seconds (reference range 28.9-38.1 seconds), a factor VIII level of $2 \%$ (reference range $50-150 \%$ ), and a mixing study diagnostic of a factor VIII inhibitor. The Bethesda Units at that time were 14.7. Her $\mathrm{CBC}$ and complete metabolic profile (CMP) were unremarkable. She was given intravenous immunoglobulin, recombinant factor VIIa at a dose of $90 \mathrm{mcg} / \mathrm{kg}$ followed by five days of recombinant factor VIII. No improvement in factor VIII level was observed. However, she achieved a good clinical response (stable ecchymotic lesion) without the need for additional recombinant Factor VIIa, and was discharged home on prednisone $100 \mathrm{mg}$ daily with a three-day taper to $60 \mathrm{mg}$ daily, and cyclophosphamide $50 \mathrm{mg}$ daily.
Over the next 20 days the patient developed six spontaneous bleeding events involving bilateral hands, elbow, tongue, cheek, and lower back. She received escalating doses of cyclophosphamide and prednisone with each acute bleed and required recombinant factor VIIa to achieve hemostasis, and additional intravenous immunoglobulin (IVIG) with the last bleeding event. As she was considered refractory to first line treatment, she began treatment with rituximab weekly for four weeks.

The patient developed two more spontaneous bleeding events during the first week of rituximab therapy. She continued with weekly rituximab therapy and has since achieved hemostatic control without subsequent bleeding events. She was eventually tapered off the immunosuppressive medications, and continues to remain symptom free for over one year. Her asthma has remained well-controlled to date on inhaled corticosteroids and montelukast.

Over the course of her presentation with the acquired inhibitor, her factor VIII activity level had reached $0 \%$, her aPTT had risen as high as 100.6 seconds, and Bethesda Units as high as 20 . Thirty-one days following commencement of rituximab therapy, her factor VIII activity rose to $23 \%$ and her Bethesda Units dropped to 1.5. Ten months after completion of rituximab, her aPTT was measured at 32 seconds and factor VIII activity level within normal limits at $84 \%$.

\section{Discussion}

Factor VIII is the most targeted clotting factor by an autoantibody, which can lead to the bleeding disorder hemophilia A. The development of factor VIII inhibitors is often idiopathic, although it has been identified in numerous conditions including malignancy, the peripartum period, autoimmune and cutaneous disorders, graftversus-host disease following bone marrow transplant, and with the use of medications such as penicillin and phenytoin [1]. It has also been described in the literature to develop in association with asthma, although the incidence of this is poorly defined [1-4]. We were unable to identify a direct relationship between the medication list of our patient and acquired hemophilia at the time of this publication.

The elderly are most often affected by acquired hemophilia A,

*Corresponding author: Askin D, Department of Hematology and Oncology, Lenox Hill Hospital, NY, USA, E-mail: davidaskin@aol.com

Received May 10, 2012; Accepted May 29, 2012; Published May 31, 2012

Citation: Askin D, Baskin M, Levine RL (2012) Spontaneous subcutaneous and muscular bleeding due to Acquired Hemophilia a in Association with PoorlyControlled Asthma: A Case Report. J Blood Lymph 2:107. doi:10.4172/21657831.1000107

Copyright: (C) 2012 Askin D, et al. This is an open-access article distributed under the terms of the Creative Commons Attribution License, which permits unrestricted use, distribution, and reproduction in any medium, provided the original author and source are credited. 
although it can occur at any age [4]. The incidence is approximately 1 to 4 per million/year [5]. The incidence in men and women is similar except in the age range 20-40 when the effect of pregnancy results in preponderance in women [5].

Evidence of the acquired inhibitor may first be detected on routine coagulation studies as an incidental finding of a prolonged aPTT. More commonly patients endure significant morbidity and mortality from this condition, as is true for the patient presented above. The bleeding is typically dissimilar from congenital hemophilia A in that hemarthroses are rare. Patients will typically present with subcutaneous bruising, mucosal and soft tissue bleeds such as intracranial hemorrhage, muscle bleeds and retroperitoneal hematoma [6].

The diagnosis of an acquired factor VIII inhibitor begins with standard coagulation studies, most commonly demonstrating a prolonged aPTT with a normal PT. Mixing studies may show correction of the prolonged aPTT, although the effect of the inhibitor can be demonstrated by incubation of the aPTT. Incubated plasma will result in a prolonged aPTT, within two hours, as the antibody is time and temperature dependent. Confirmation of a factor VIII inhibitor is made by a low factor VIII and an elevated inhibitor titer on Bethesda assay [6].

Management of bleeding typically involves two separate but distinct concepts; achieving hemostasis in the setting of acute bleeding, and eliminating the antibody via immune suppression. Both activated prothrombin complex concentrate (FEIBA), and recombinant factor VIIa have proven effective in control of acute bleeding episodes in the majority of acquired hemophilia cases. DDAVP can be considered in the control of minor non-life threatening bleeding if the level of inhibitor is low [7].

Eradication of the inhibitor is required to minimize the morbidity and mortality associated with acquired hemophilia A, and to provide long-term hemostasis. While some inhibitors will spontaneously resolve, it is difficult to identify these patients, and therefore all patients diagnosed with acquired hemophilia A should be considered for immunosuppression at the time of diagnosis. Frontline therapy including corticosteroids (prednisone) and alkylating agents (cyclophosphamide) are typically effective in eliminating the circulating antibody, however refractory cases may require additional therapy. Rituximab, a monoclonal antibody to CD20+ B-cells has proven effective in transiently eliminating the inhibitor in acquired hemophilia A patients, with reported response periods ranging upwards of 30 months in responders [8-9].

\section{Conclusion}

Acquired hemophilia A is a condition with significant morbidity, including limb-threatening complications. It can be idiopathic, or associated with an autoimmune disorder such as rheumatoid arthritis or systemic lupus erythematosis, and has been shown in the literature to occur in patients with asthma. Effective treatment involves achieving hemostasis and eradicating the inhibitor. Options for eradication include immunosuppressive agents and rituximab therapy.

It is important to consider this diagnosis in asthmatics that present with alterations in coagulation studies (aPTT) or those with spontaneous bleeds, as rapid recognition and treatment can limit the morbidity and mortality associated with this dangerous condition.

\section{References}

1. Sohngen D, Specker C, Bach D, Kuntz BME, Burk M, et al. (1997) Acquired Factor VIII inhibitors in nonhemophilic patients. Ann Hematol 74: 89-93.

2. Bossi P, Cabane J, Ninet J, Dhote R, Hanslik T, et al. (1998) Acquired hemophilia due to factor VIII inhibitors in 34 patients. Am J Med 105: 400-408.

3. del Campo R, Bargay J, Cladera A, Balaguer H, Requena MJ, et al. (2010) Acquired hemophilia $A$ : three different presentations of the same disease. Blood Coagul Fibrinolysis 21: 188-191.

4. Boggio Lisa N, Green David (2001) Acquired Hemophilia. Rev Clin Exp Hematol 5: 389-404.

5. Franchini Massimo, Lippi Giuseppe (2008) Acquired factor VIII inhibitors. Blood 112: $250-255$

6. Collins PW (2011) Management of acquired hemophilia A. J Thromb Haemost 9: 226-235.

7. Hay Charles RM, Brown S, Collins PW, Keeling DM, Liesner R (2006) The diagnosis and management of factor VIII and IX inhibitors: a guideline from the United Kingdom Haemophilia Centre Doctors Organisation. Br J of Haematol 133: $591-605$

8. Wiestner Adrian, Cho Hearn J, Asch Adam S, Michelis Mary Ann, Zeller Jack A et al. (2002) Rituximab in the treatment of acquired factor VIII inhibitors. Blood 100: 3426-3428

9. Field JJ, Fenske TS, Blinder MA (2007) Rituximab for the treatment of patients with very high-titre acquired factor VIII inhibitors refractory to conventional chemotherapy. Haemophilia 13: 46-50. 